\title{
Transmission Electron Microscopy of Crystallization of Lysozyme in a Solution
}

\author{
Tomoya Yamazaki ${ }^{1}$, Manabu Shirai ${ }^{2}$, Hiroaki Matsumoto ${ }^{2}$ and Yuki Kimura ${ }^{1}$ \\ 1. Institute of Low Temperature Science, Hokkaido University, Sapporo, Japan \\ 2. Hitachi High-Technologies Corporation, Hitachinaka, Japan
}

It is important to observe the crystallization process of proteins as the fundamental study of the material science and for the structure analysis of a protein molecule by X-ray diffraction pattern using a protein crystal. The useful method to study the crystallization process is in-situ microscope observation because it can directly visualize the real process. The observation of protein crystallization have been performed mainly using optical microscopy [1,2] and the micro-scale view of the process have been well understood. However, the protein crystallization process at nano-scale is still unclear because there is no in-situ observation of it. Recently, in-situ observations of the behavior of nano-particles and the crystallization processes of inorganic materials have been energetically performed by the transmission electron microscopy (TEM) combined with the liquid cell or an ionic liquid, and these mechanisms at nano-scale are partly demonstrated [3-5]. However, there are no observations of crystallization of proteins so far. We performed in-situ observation of the protein crystal for understanding its crystallization process using TEM with the liquid cell.

We used the hen-egg white lysozyme as a protein sample. The lysozyme was crystallized using $\mathrm{NaCl}$ as a precipitant in a sodium acetate buffer solution at $\mathrm{pH}=4.5$. For the observation, we used a "Poseidon" liquid cell holder (Protochips Inc.) with two input and one output ports. The liquid cell consists of a pair of semiconductor-based plates with an amorphous silicon nitride window and 150 or 500-nm-thick spacer to form the flow path of the crystallization solution. We used two TEMs with LaB 6 filament at an acceleration voltage of $200 \mathrm{kV}$ (Hitachi H-8100) and with field-emission gun at an acceleration voltage of $300 \mathrm{kV}$ (Hitachi HF-3300).

We succeeded in observing the two crystalline phases of orthorhombic and tetragonal in addition to an amorphous phase of the lysozyme protein. Individual amorphous particles could not observe under optical microscopy because the size of the amorphous particles is only about 100-200 nm (Fig. 1), which is smaller than the spatial resolution of optical microscope. The growth rates of orthorhombic crystals and tetragonal crystals under TEM are consistent with those measured by optical microscopy under similar condition. Therefore, the electron-beam irradiation on the crystallization of lysozyme under TEM can be negligible in our experimental condition. We observed an orthorhombic crystal and amorphous particles in the same view. The orthorhombic lysozyme crystal grew continuously, whereas amorphous particles dissolved slowly. It clearly shows that the solubility of an orthorhombic crystal is lower than that of amorphous particles, i.e., the orthorhombic lysozyme crystal is more stable than the amorphous particles of lysozyme. As a conclusion, we succeeded in observing the nano-scale view of the protein crystallization using the TEMs and the liquid cell holder as a first step for understanding of the protein crystallization process [6]. 


\section{References}

[1] P. Dold et al., J. Cryst. Growth 293 (2006), 102.

[2] A. E. S. Van Driessche et al., Cryst. Growth \& Des. 7 (2007), 1980.

[3] J. M. Yuk et al., Science 336 (2012), 61.

[4] Y. Kimura et al., J. Am. Chem. Soc. 136 (2014), 1762.

[5] M. H. Nielsen et al., Science 345 (2014), 1158.

[6] The authors acknowledge supports from a Grant-in-Aid for Research Activity Start-up from KAKENHI (26887001) and for a grant for Young Scientists (A) from KAKENHI (24684033).

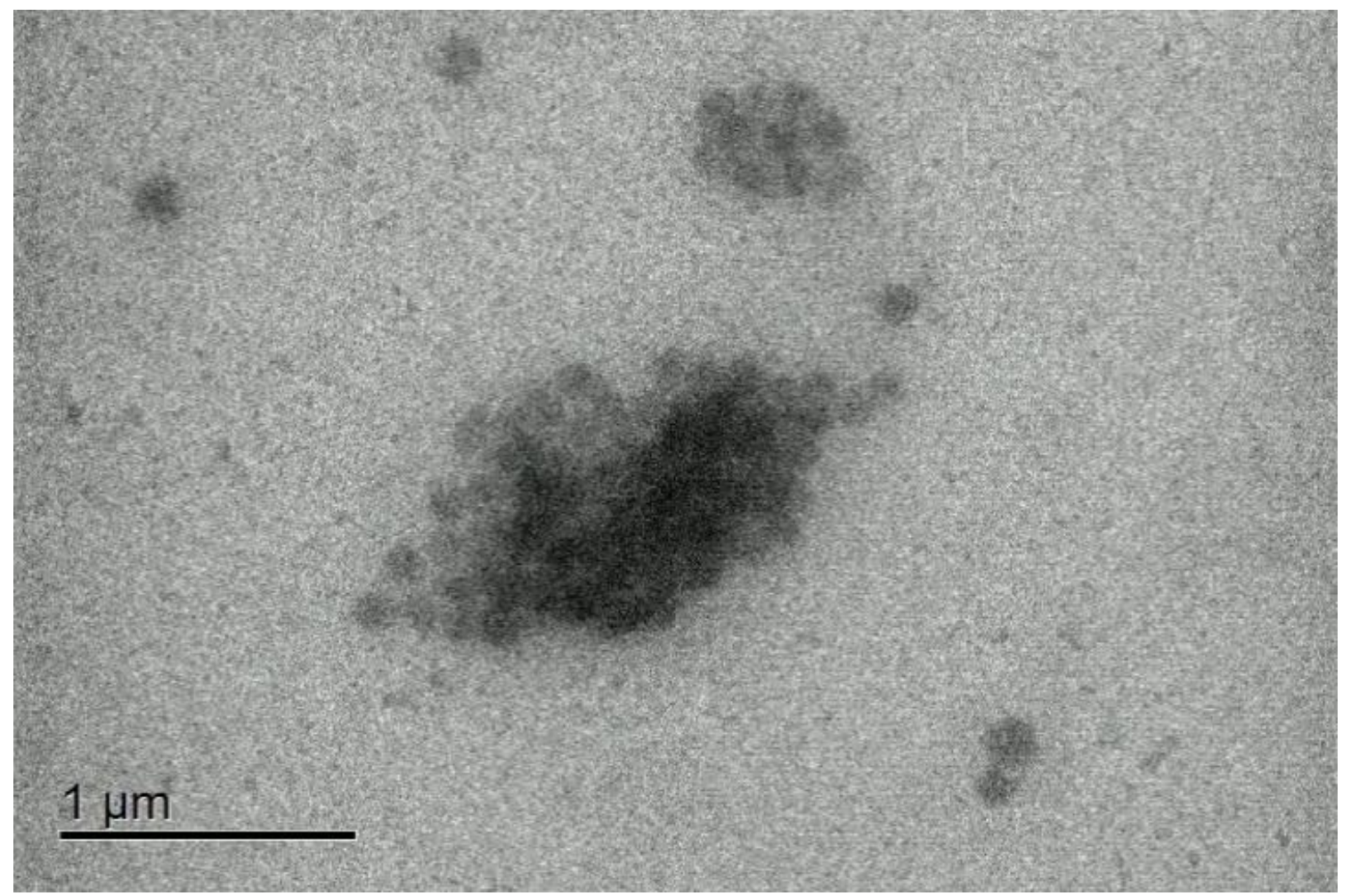

Figure 1. A bright-field TEM image of a cluster of lysozyme amorphous particles in a solution. This image was recorded by a Hitachi HF-3300 with an acceleration voltage of $300 \mathrm{kV}$. The size of an individual particle is about $100-200 \mathrm{~nm}$. 